\title{
Holistic practice and excessively thorough paperwork: A response to Julião et al.
}

\section{Letter to the Editor}

Cite this article: Kingdon A, Spathis A, Barclay S (2020). Holistic practice and excessively thorough paperwork: A response to Julião et al. Palliative and Supportive Care 18, 632-633. https://doi.org/10.1017/S1478951520000851

Received: 17 August 2020

Accepted: 23 August 2020

Author for correspondence: Arjun Kingdon, Department of Public Health and Primary Care, University of Cambridge, Institute of Public Health, Forvie Site, Cambridge Biomedical Campus, Cambridge CB2 OSR, UK. E-mail: adnk2@medschl.cam.ac.uk
Arjun Kingdon, M.A., M.B., B.CHIR, M.R.C.P. (D), Anna Spathis, M.A., M.B., B.CHIR., M.D., F.R.C.P., M.R.C.G.P., F.H.E.A. and Stephen Barclay, M.A., B.M., B.C.H., M.SC., F.R.C.G.P., F.H.E.A., M.D.

Department of Public Health and Primary Care, University of Cambridge, Institute of Public Health, Cambridge, UK

Dear Editor,

We read Julião et al.'s recently published paper (Julião et al., 2020) with interest. Our experiences, working in palliative care services in the UK, are consistent with the authors' findings of more limited documentation of psychosocial than physical needs in initial palliative care assessments. This may result from the ongoing primacy of the medical model, even in palliative care settings where holism is promoted. In an attempt to drive a comprehensive holistic needs assessment, many palliative care services have developed detailed paperwork to support clerking on admission to an inpatient unit. While this may be particularly useful as an aidememoire for junior staff and those less familiar with the holistic approach of palliative care, it is our experience that, in practice, psychosocial needs are frequently dxeprioritized due to the existing culture of emphasizing the exploration of physical symptoms. Additionally, given the ubiquity of fatigue in patients with palliative care needs (Mücke et al., 2015), comprehensiveness of information gathering at an initial meeting must be balanced against the risk of exhausting the patient. Over-emphasis on thoroughness at a first meeting risks sapping the patient's energy and goodwill and often it is the psychosocial issues that are initially explored to a lesser degree.

The limited amount of existing evidence concerning the impact of admission paperwork on clerking outcomes focuses on efforts to increase thoroughness through the use of proformas (Chow et al., 2014; Ehsanullah et al., 2015; Smallwood et al., 2018), demonstrating increased completeness when proformas are used, but with no measurement of patient-centered impact or outcomes. While some prompts are undeniably useful, holism may at times be discouraged by overly prescriptive paperwork that aims for absolute thoroughness.

We propose that if holistic needs assessment education was embedded at an earlier stage of clinical experience, particularly undergraduate medical training, organizations would be able to place greater reliance on the judgement of their staff, including non-specialist junior doctors. This could result in redesign of clerking paperwork to emphasize holistic practice and patients' needs, rather than rigid tools designed around healthcare professionals' agendas. Doctors would have the skills to recognize patients' level of fatigue, enabling them to prioritize initially obtaining the information needed to enable them to address individual patients' main priorities and most pressing needs, while also practicing safely. Subsequent consultations would allow the multidisciplinary team to "fill in the gaps". This would avoid the imposition of institutionally predetermined priorities, mediated through having to meet the needs of a proforma, interfering with a genuinely patient-led initial clerking consultation.

Paperwork can and should encourage holistic practice, although for safety-minded organizations, taking a leap away from inflexible and prescriptive paperwork is challenging. Widespread implementation of early education about patient-centered consultations, reinforced through supervised early practice, would enable this leap to be successful and patient care to be ultimately more holistic.

Authorship. A.K. wrote the manuscript with editing and supervisory input from S.B. and A.S. All authors have read and approved the manuscript.

Funding. The author(s) disclosed receipt of the following financial support for the research, authorship, and/or publication of this article: A.K. is funded by a Health Education East of England Academic Clinical Fellowship. S.B. is funded by the National Institute for Health Research (NIHR) Applied Research Collaboration East of England (ARC EoE) programme. The views expressed are those of the author(s) and not necessarily those of the NHS, the NIHR or the Department of Health and Social Care.

Conflict of interest. The authors declared that they have no conflict of interest.

\section{References}

Chow J, Yvon C and Stanger T (2014) How complete are our clerkings? A project aimed at improving the quality of medical records by using a standardised proforma. BMJ Quality Improvement Reports 2(2), u203012.w1388. doi:10.1136/bmjquality.u203012.w1388. 
Ehsanullah J, Ahmad U, Solanki K, et al. (2015) The surgical admissions proforma: Does it make a difference? Annals of Medicine and Surgery 4(1), 53-57. doi:10.1016/j.amsu.2015.01.004.

Julião M, Sobral MA, Calçada P, et al. (2020) “Truly holistic?” Differences in documenting physical and psychosocial needs and hope in Portuguese palliative patients. Palliative and Supportive Care, 1-6. doi:10.1017/ S1478951520000413.
Mücke M, Mochamat M, Cuhls H, et al. (2015) Pharmacological treatments for fatigue associated with palliative care. Cochrane Database of Systematic Reviews 5, CD006788. doi:10.1002/14651858.CD006788.pub3.

Smallwood N, Russell J, Forbes-Pyman R, et al. (2018) Do medical admission pro formas improve the completeness of documentation? A multisite observational study. Future Healthcare Journal 5(2), 121-125. doi:10.7861/futurehosp.5-2-121. 